\title{
Forefront of $\mathrm{Na}^{+} / \mathrm{Ca}^{2+}$ Exchanger Studies: Preface
}

\author{
Takahiro Iwamoto ${ }^{1, *}$ \\ ${ }^{1}$ Department of Pharmacology, School of Medicine, Fukuoka University, Fukuoka 814-0180, Japan
}

Received June 14, 2004; Accepted July 27, 2004

Keywords: $\mathrm{Na}^{+} / \mathrm{Ca}^{2+}$ exchanger (NCX), structure and function, $\mathrm{Ca}^{2+}$ signaling, transport mechanism, $\mathrm{NCX}$ inhibitor

This Forum Minireview contains the proceedings of the symposium at the 76th Annual Meeting of The Japanese Pharmacological Society (March 24, 2003). The aim of the symposium was to summarize recent advances in our understanding of the structure and function of the $\mathrm{Na}^{+} / \mathrm{Ca}^{2+}$ exchanger (NCX) family in heart, smooth muscle cells, neurons, and nephron cells. NCX is a plasma membrane transporter that is expressed in many mammalian cell types. This exchanger is bidirectional and is regulated by membrane potential and transmembrane gradients of substrate ions. This issue presents the steady achievements of developing experimental tools, such as selective NCX inhibitors and NCX-engineered mice and electrophysiological advances in the study of NCX. It is our hope that our descriptions of these recent advances will provide a valuable resource in which readers may find clues to solve the puzzle of the physiological and pathological functions of the NCX family.

Matsuoka shows the regulation kinetics of five NCX isoforms, NCX1, NCX2, NCX3, NCX-SQ1, and CALX. In all of the exchangers, $\mathrm{Na}^{+} / \mathrm{Ca}^{2+}$ exchange current from inside-out membrane patches are regulated by cytoplasmic $\mathrm{Ca}^{2+}$. However, the mode of regulation is different among NCX isoforms. NCX1, NCX2, and NCX-SQ1 are positively regulated by cytoplasmic $\mathrm{Ca}^{2+}$, but CALX is negatively regulated. NCX3 apparently has both positive and negative regulation mechanisms. Matsuoka finally considers the multiple mechanisms of $\mathrm{Ca}^{2+}$-dependent regulation in NCX family.

Hinata and Kimura re-examine the stoichiometry of cardiac NCX by measuring the reversal potential of the NCX current and intracellular $\mathrm{Ca}^{2+}$ concentrations under the whole-cell voltage clamp. They confirm that the stoichiometry of NCX is 3:1 not $4: 1$ and then explore the possible reasons for obtaining results supporting a

*Corresponding author. FAX: +81-92-865-4384

E-mail: tiwamoto@cis.fukuoka-u.ac.jp

\section{4:1 stoichiometry.}

Uehara et al. compare the sensitivities for monovalent cations $\left(\mathrm{Na}^{+}, \mathrm{K}^{+}\right.$, and $\left.\mathrm{Li}^{+}\right)$between $\mathrm{K}^{+}$-dependent (NCKX2) and $\mathrm{K}^{+}$-independent $\mathrm{Na}^{+} / \mathrm{Ca}^{2+}$ exchanger (NCX1) using their transfectants. They find that the sensitivities for $\mathrm{Na}^{+}, \mathrm{K}^{+}$, and $\mathrm{Li}^{+}$markedly differ between these two exchangers. NCKX2 and NCX1 are considered to be regulated differentially via the ion binding sites that have distinct sensitivities to the external monovalent cations.

Komuro and Ohtsuka present the functional importance of NCX1 on cardiac function and on ischemia /reperfusion injury using NCX1 knockout (KO) mice. The contractile function in NCX1 KO mice hearts is significantly better than that in wild type (WT) mice hearts after ischemia/reperfusion and the infracted size is significantly smaller in NCX1 KO mice hearts compared with that in WT mice hearts. They suggest that NCX1 is critically involved in the development of ischemia/reperfusion-induced myocardial injury and therefore the inhibition of NCX1 function may contribute to cardioprotection against ischemia/reperfusion injury.

Iwamoto shows the characteristics and therapeutic potentials of newly developed NCX inhibitors, KBR7943, SEA0400, and SN-6. Currently, SEA0400 is the most potent and selective inhibitor. These inhibitors display different isoform-selectivities, whereas these have similar properties such as $\mathrm{Ca}^{2+}$ influx mode-selectivity and $I_{1}$ inactivation-dependence. Recent sitedirected mutagenesis has revealed that these inhibitors possess some molecular determinants (Phe-213, Val-227, Tyr-228, Gly-833, and Asn-839) for the interaction with NCX1. These inhibitors are expected to be useful tools to study the physiological roles of NCX. Moreover, such inhibitors may offer therapeutic potential as a new remedy for ischemic disease, arrhythmias, heart failure, and hypertension. 